\title{
Correction to: Oral health literacy and oral health outcomes in an adult population in Brazil
}

\author{
Marília Jesus Batista ${ }^{1,2^{*}}$, Herenia Procopio Lawrence ${ }^{3}$ and Maria da Luz Rosário de Sousa ${ }^{4}$
}

\section{Correction}

After publication of the article [1], it has been brought to our attention that the publication funding acknowledgement is missing from the original article. It should also include reference to grant number 2017/13264-7 from the Sao-Paulo Research Foundation.

\begin{abstract}
Author details
${ }^{1}$ Community Health, Jundiai Medical School, Francisco Telles, 250, P.O. Box1295, Jundiaí SP 13202-550, Brazil. ${ }^{2}$ Community Health Graduate Program, Piracicaba Dental School, University of Campinas, Avenida Limeira 901, P.O. Box 52, Piracicaba SP 13414-018, Brazil. ${ }^{3}$ Dental Public Health Discipline, Faculty of Dentistry, University of Toronto, Canada, 124 Edward Street, Toronto ON M5G 1G6, Canada. ${ }^{4}$ Department of Community Dentistry, Piracicaba Dental School, University of Campinas, Brazil, Avenida Limeira 901, P.O. Box 52, Piracicaba SP 13414-018, Brazil.
\end{abstract}

Received: 4 October 2017 Accepted: 5 October 2017

Published online: 18 October 2017

\section{Reference}

1. Batista M, Lawrence $H$, Sousa M. Oral health literacy and oral health outcomes in an adult population in Brazil. BMC Public Health. 2017;18(1):60. https://doi.org/10.1186/s12889-017-4443-0

\footnotetext{
* Correspondence: mariliajbatista@yahoo.com.br

1 Community Health, Jundiai Medical School, Francisco Telles, 250, P.O. Box1295, Jundiaí SP 13202-550, Brazil

${ }^{2}$ Community Health Graduate Program, Piracicaba Dental School, University of Campinas, Avenida Limeira 901, P.O. Box 52, Piracicaba SP 13414-018, Brazil
} 